\title{
On Acquisition of Wideband Direct-Sequence Spread Spectrum Signals
}

\author{
Iyappan Ramachandran, Student Member, IEEE and Sumit Roy, Member, IEEE
}

\begin{abstract}
Very high rate packet data systems such as those based on wideband spread-spectrum (SS) signaling face an important challenge - achieving fast and reliable acquisition to minimize preamble overhead and optimize packet throughput. Such wideband modulation schemes undergo frequency selective fading, implying a very large number of resolved multipath components. Thus, the conventional analysis of serial search acquisition schemes based on simplifying assumptions that are valid in narrow(er) band systems (and are invalid for sufficiently wideband systems) need to be revamped. Also, there is a need to devise new schemes that exploit the presence of multipath components for faster acquisition over simple serial search. Our work provides novel contributions in both these aspects: we analyze serial and random search acquisition schemes and compare their performances in several multipath environments. It is shown that over many typical indoor channels, random search offers significantly lower mean acquisition times.
\end{abstract}

Index Terms-Acquisition, wideband signals, multipath, serial search, random search, ultra-wideband.

\section{INTRODUCTION}

Code synchronization in direct-sequence spread spectrum systems is the process of aligning the receiver's locally generated code with that in the received signal. Successful code synchronization is fundamental to a successful spread spectrum receiver since it precedes despreading and demodulation. Code synchronization is typically done in two stages: code acquisition for coarse alignment and code tracking for fine synchronization; the former is the focus of this paper.

In packet based wireless systems, code synchronization is done on a per packet basis, typically requiring a known preamble within the packet header for synchronization purposes. As packet based systems strive to achieve very high data throughput, the preamble should be kept as small as possible to minimize overhead, i.e. the need is to achieve fast acquisition/synchronization. The problem is compounded by the severe multipath environments that these high data rate systems typically operate in. In fact, reducing the (mean) acquisition time while maintaining the reliability of acquisition/synchronization in multipath is probably the single most significant challenge in high data rate SS system design.

Initial development and analysis of acquisition schemes ([1] and [2], for example) were conducted for the case where there exists only one resolvable path and consequently only one $H_{1}$

This work was supported in part by the National Science Foundation under Grant ANI 0325014 (ITR: RESCUENET-Embedded In-Building Sensor Network to Assist Disaster Rescue).

The authors are with the Dept. of Electrical Engineering, University of Washington, Seattle, WA 98195, USA. Email: \{iyappan, roy\}@ee.washington.edu (in-phase or target present) cell. Subsequent work has focussed on the effect of the presence of multiple $H_{1}$ cells. For example, [3] investigates mean acquisition time (MAT) of serial search for the case when the search starts outside the $H_{1}$ cells. In [4], the multiple $H_{1}$ cells are replaced with a single $H_{1}$ cell with an equivalent $P_{D}$. These assumptions and the resulting analysis are valid when the number of $H_{1}$ cells, $L$ is much smaller than the total number of cells, $q$ that an acquisition receiver has to test, characteristic of DS-SS systems using long PN codes. In [5], serial search was analyzed for an $L$-path case but with the assumption of equal powers. To the author's knowledge, there does not exist a comprehensive analysis of the serial search scheme in general multipath scenarios without simplifying assumptions. Such an analysis would be important when either short codes are employed or when the number of multipath components are very large due to the fine resolution of the signals used, i.e., $L<<q$ is not necessarily valid, and constitutes a notable contribution of our work.

Further, serial search is best suited for dense channels, i.e. where a multipath arrival is present at every tap in a delay line representation of the equivalent discrete channel. However, several indoor channels exhibit a certain sparseness since the multipath arrivals occur in clusters. The channel model adopted by the IEEE 802.15.3a for UWB propagation [6], [7], for example, uses two Poisson distributions to characterize multipath arrivals. This inherent randomness in multipath arrivals has prompted considerable interest in random search as a possible scheme for faster acquisition. To the authors' knowledge, random search acquisition has not been analyzed in the literature with the exception of [8], where only the 'ideal' case of zero false alarm probability and unity detection probability is considered. We conduct an analysis of random search leading to the derivation of a compact formula for the MAT.

An accompanying motivation for our work is provided by the recent upsurge of interest in ultra-wideband systems [9], [10], [11] which are characterized by bandwidths in excess of $500 \mathrm{MHz}$ resulting from the use of appropriately shaped, ultra-thin pulses for transmission. The resulting fine time resolution leads to a large number of significant multipath components. Hence, we have chosen ultra-wideband channel models adopted by 802.15.3 standards group in addition to the conventional wideband channel models for evaluation of acquisition schemes and performance comparisons.

In summary, the main contributions are: (a) accurate analysis of serial and random searches over general wideband multipath channel models, and (b) performance comparison of serial and random searches in wideband channels and 
802.15.3a channels. As a further contribution, we consider a non-consecutive search scheme presented in [12] and compare its performance with serial and random searches in conventional wideband channel models. It is worth mentioning that while the analytical approach developed for serial search is not applicable to the 802.15.3a channel models (in which the assumption of multipath arrival at every channel tap made in the analysis does not hold), the random search analysis makes no assumption about the channel model and can be applied to all channel models.

\section{SySTEM DESCRIPTION}

\section{A. Signal model}

For packetized data transmission with conventional DSSS modulation with short spreading codes, each bit is sent by a length $N_{c}$ PN sequence of \pm 1 binary chips that are periodically repeated in each bit interval. However, during the preamble-assisted acquisition phase, it is assumed that an unmodulated PN sequence is transmitted, i.e.,

$$
s(t)=\sqrt{E_{c}} \sum_{k} c_{k} p\left(t-k T_{c}\right)
$$

where $\left\{c_{k}\right\}$ is the pseudo-random code sequence and the energy in the pulse $p(t)$ is normalized to unity (hence, $E_{c}$ is the transmitted energy per chip). For the specific case of UWB modulation based on direct sequence spread spectrum (DSUWB) as embodied in a proposal before the IEEE 802.15.3a standard body [13], an appropriately shaped baseband pulse shape is used. We use the $2^{\text {nd }}$ derivative of a Gaussian pulse [8] in contrast to raised cosine pulses used in [13] primarily for the known analytical benefits.

$$
p(t)=\sqrt{\frac{4}{3 t_{n} \sqrt{\pi}}}\left(1-\left(\frac{t}{t_{n}}\right)^{2}\right) \exp \left(-\frac{1}{2}\left(\frac{t}{t_{n}}\right)^{2}\right)
$$

The parameter $t_{n}$ determines the effective time width of the
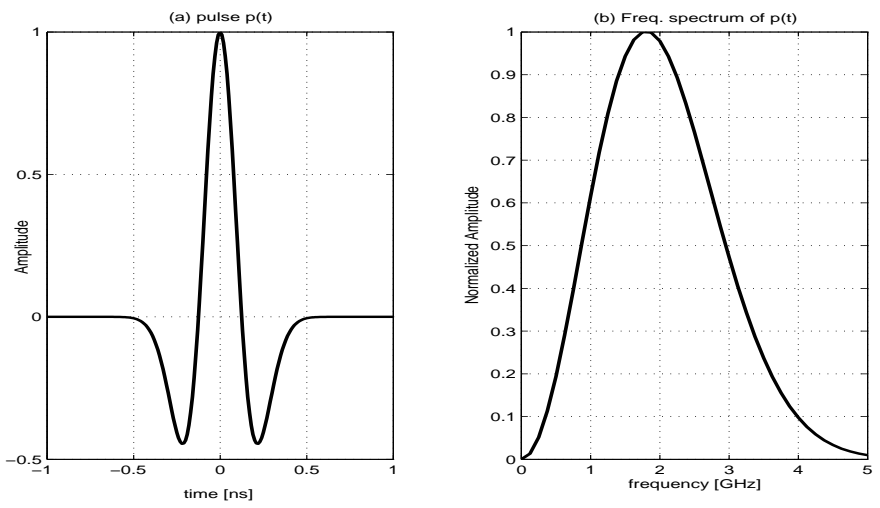

Fig. 1. (a) The pulse $p(t)$ as a function of time (b) The normalized magnitude spectrum of $p(t)$

pulse $T_{p}$ and hence its bandwidth. The pulse $p(t)$ and its frequency spectrum are shown in Fig. 1 for $t_{n}=0.125 \mathrm{~ns}$. The effective time duration of the pulse for this value of $t_{n}$ is $T_{p} \approx 1 \mathrm{~ns}$ and the $10-\mathrm{dB}$ bandwidth is approximately 4 $\mathrm{GHz}$. The chip duration has been assumed to be equal to the pulse duration, i.e. $T_{c}=T_{p}$. Note that there is no explicit upconversion (carrier modulation) stage at the transmitter, and hence DS-UWB modulation can be simply treated as an equivalent baseband signal.

Gaussian shaped pulses have the flexibility of spectral shaping (to meet the FCC specified mask, for example) by appropriate choices of the derivative order and pulse parameters. In traditional wideband DS-SS, bandwidth scaling is achieved primarily via reducing the chip duration and the effect of the pulse shape is largely secondary. In UWB systems however, pulse shaping significantly affects the system bandwidth. In whichever manner bandwidth scaling is achieved, the objective of this paper is to investigate the effect of such wide bandwidths on the acquisiion performance. It is worth mentioning that the particular choice of the pulse shape changes only the signal bandwidth and hence the multipath resolution, but does not affect the analysis carried out in this paper.

\section{B. Channel models}

We consider two different wideband channel models - the first is the conventional channel model based on an $L$-tap delay line with a multipath component at each delay and the second is that adopted by the IEEE 802.15.3a task group based on the Saleh-Valenzuela model.

1) Conventional tapped delay line model: In a timeinvariant multipath channel, the received signal is given by

$$
s_{\text {rec }}(t)=s(t) * h(t)+n(t)
$$

where $h(t)$ is the impulse response of the channel and $n(t)$ is a zero mean additive white Gaussian noise process with a power spectral density of $N_{0}$. The wideband channel can be represented by a tapped delay-line model with the spacing of a minimum multipath resolution $t_{s}$, that is determined by the bandwidth of the system. For convenience, we set $t_{s}=T_{p} / M$, where $T_{p}$ is the pulse duration and $M$ is a positive integer.

$$
h(t)=\sum_{l=1}^{L} \alpha_{l} \delta\left(t-(l-1) t_{s}\right)+n(t)
$$

where $L$ denotes the number of resolvable paths. In our analysis, we assume an exponentially decaying multipath intensity profile $\left(E\left[\alpha_{l}^{2}\right]\right.$ versus the lag $\left.l\right)$ with a decay factor of $\eta$; this was assumed as the model for indoor channels in the $2.45 \mathrm{GHz}$ ISM band by the standards group for IEEE $802.11 \mathrm{~b}$ Wireless LANs. When the total power in all the resolvable paths is normalized to unity, the (average) power in the $l$-th path is expressed as

$$
E\left[\alpha_{l}^{2}\right]=\frac{1-e^{-\eta}}{1-e^{-\eta L}} e^{-(l-1) \eta}
$$

In wideband channels as in UWB, the number of unresolved multipath components per tap are expected to be limited due to the fine time-resolution. Therefore the Rayleigh distribution, widely used for narrowband systems, is not a suitable model for the tap weight distribution; instead, independent lognormal distributions has been found to be a good fit from some experimental wideband channel measurements [6]. Further, it is important to note that the channel coefficients are assumed 
real and not complex since we assume that the wideband transmission is a baseband signal [7] (there is no explicit frequency upconversion/downconversion as in conventional narrowband systems).

2) IEEE 802.15.3a channel model: Many indoor channels display a certain 'sparseness' when modelled as a delay line the component multipaths are typically clustered. The model adopted by the IEEE 802.15.3a for UWB propagation in 3$10 \mathrm{GHz}$ band [6] is a (frequency selective) delay line based on the celebrated Saleh-Valenzuela (S-V) model [14]. This is characterized by two Poisson distributions for the arrival times - one for the delay of the first path of each cluster $T_{l}$ with a mean (cluster) arrival rate of $\Lambda$; the second for the paths within each cluster $\tau_{k, l}$ with a mean (path) arrival rate of $\lambda$. The distributions of the cluster and path arrival times are thus given by

$$
\begin{aligned}
p\left(T_{l} \mid T_{l-1}\right) & =\Lambda \exp \left[-\Lambda\left(T_{l}-T_{l-1}\right)\right] \\
p\left(\tau_{k, l} \mid \tau_{(k-1), l}\right) & =\lambda \exp \left[-\lambda\left(\tau_{k, l}-\tau_{(k-1), l}\right)\right]
\end{aligned}
$$

Therefore, the multipath channel has the following discrete time impulse response.

$$
h(t)=\sum_{l=0}^{L-1} \sum_{k=0}^{K-1} \beta_{k, l} \delta\left(t-T_{l}-\tau_{k, l}\right)
$$

where $\beta_{k, l}$ are the real multipath gain coefficients corresponding to a baseband model. The multipath intensity profile is defined by a double exponential decay model

$$
E\left[\beta_{k, l}^{2}\right]=\Omega_{0} e^{-T_{l} / \Gamma} e^{-\tau_{k, l} / \gamma}
$$

where $\Omega_{0}$ is the mean power of the first path of the first cluster and $\Gamma$ and $\gamma$ are the cluster and path decay factors respectively. The amplitudes are assumed to be independent log-normal distributions.

\section{Structures of the acquisition receivers}

We consider four different acquisition receiver structures in this work. The first three - serial search, random search and non-consecutive search with cell-by-cell detection - can be represented by the basic structure shown in Fig. 2, each differing only in the search logic employed, while the fourth, the non-consecutive search receiver with joint twin-cell detection receiver is depicted in Fig. 3.

1) Serial search acquisition receiver: The conventional method used to synchronize the received signal with the receiver's local code is the serial search scheme using the sliding correlator [15]. The receiver works by shifting the local code progressively in steps of size $t_{s}$ equal to the multipath resolution. At every shift position (also called a cell), a decision variable is formed by correlating the local code with the received code over a correlator dwell time, which in our case has been assumed to be one full period of the PN code, i.e. $\tau_{D}=N_{c} T_{c}$ seconds. The decision variable is then compared with a decision threshold $T_{h}$. If the decision variable exceeds the threshold, the corresponding cell is tentatively declared to be an in-phase or $H_{1}$ cell (signal present) and the system goes into a verification mode. The result of the verification stage may be a confirmation of the $H_{1}$ cell, in which case acquisition is declared to have been achieved and the search terminated; alternatively, a false alarm is detected, in which case the search resumes after a fixed penalty time of $J \tau_{D}$ seconds. If the threshold is not exceeded, the corresponding cell is declared to be an out-of-phase cell ( $H_{0}$ cell) and the receiver proceeds to test the next code shift. The process is repeated until acquisition which is defined as the state when the receiver locks on to one of the $H_{1}$ cells to within a step size ambiguity. The number of cells in an uncertainty region of $N_{c} T_{c}$ seconds is thus $q=N_{c} T_{c} / t_{s}=N_{c} M$.

2) Random search acquisition receiver: The random search receiver is very similar to the serial search receiver except that the search logic is to shift the phase of the local code by a random multiple of $t_{s}$ chosen between 0 and $(q-1)$. The receiver continues to run with random jumps in local code phase at each step until acquisition is achieved. The observation that the MAT for random search may be significantly lower as compared to serial search in some profiles when the number of multipath components is large provides a compelling case to study the random search receiver.

3) Non-consecutive search acquisition receiver with cellby-cell detection (NCS-CC): The non-consecutive search scheme was proposed in [12], where the scheme has been applied and analyzed for the acquisition of conventional DSSS signals. In order to exploit the presence of multiple $H_{1}$ cells, the search logic is modified such that the correlator step size is $L t_{s}$ durations, where $L$ denotes the number of resolvable paths assumed known to the receiver. In the conventional tapped delay-line model with a multipath component at every tap position, the NCS scheme ensures that the $H_{1}$ cells are uniformly distributed in the uncertainty region. This reduces the mean time to reach a $H_{1}$ cell from an initial cell. An additional phase adjustment by one $t_{s}$ duration is required in the search logic after every $N_{c} T_{c} / L t_{s}=N_{c} M / L$ steps to avoid testing the same cells over again in the next cycle and ensure that all the cells in the uncertainty region are searched.

4) Non-consecutive Search Acquisition Receiver with Joint Twin-Cell Detection (NCS-TC): In wideband systems, the received signal energy is spread among many multipath components. It seems reasonable then to expect that a search scheme that utilizes energy over multiple paths would perform better than the one that does not, i.e. bases it's decision only on isolated cells. A joint twin-cell detection scheme has been proposed in [3], where the decision variable is formed by combining detector outputs corresponding to two successive cells; clearly the price is increased hardware cost due to the need for a pair of correlators. To avoid the problem of correlation between successive decision variables, we implement the joint twin-cell scheme with non-consecutive search as suggested in [12], where a joint triple-cell detection scheme is employed. The structure of the acquisition receiver employing NCS-TC is shown in Fig. 3. The receiver consists of a pair of correlators that are supplied by the same code generator; one of the inputs is however is delayed with respect to the other by one step size $t_{s}$. The outputs of the two correlators, $y_{1}$ and $y_{2}$ are then added non-coherently with equal weights to form the decision variable $y$. If the two cells being combined are $H_{1}$ cells, the combined output $y$ has a higher energy than that obtained 


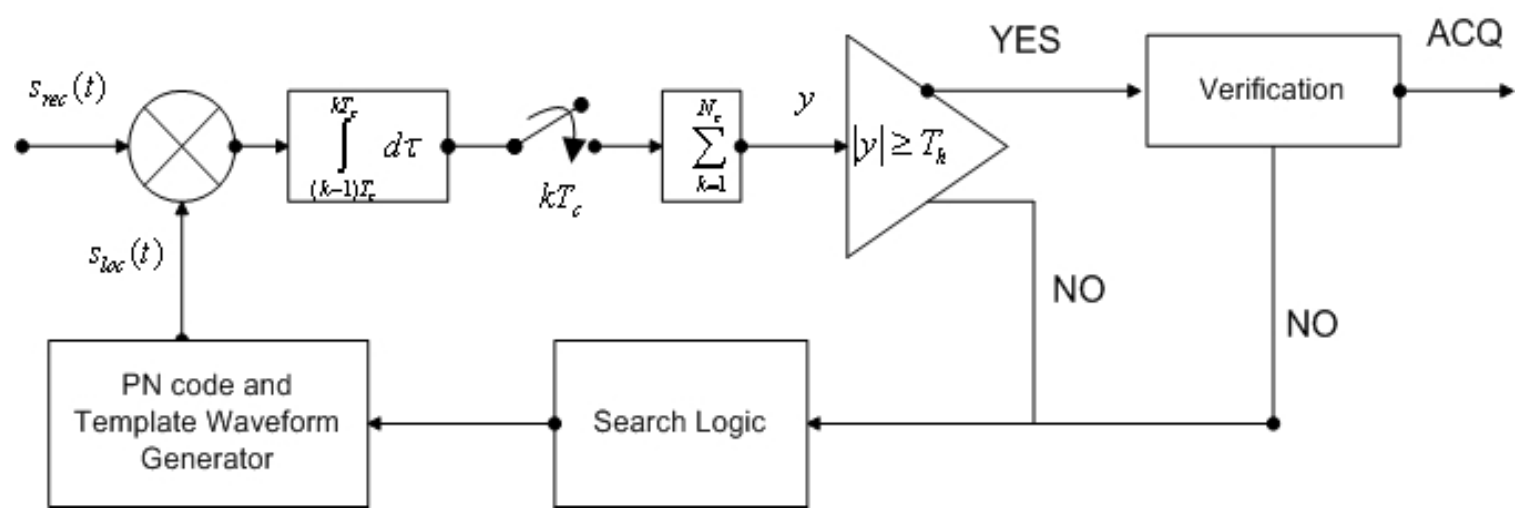

Fig. 2. General structure of serial, random and NCS acquisition receivers

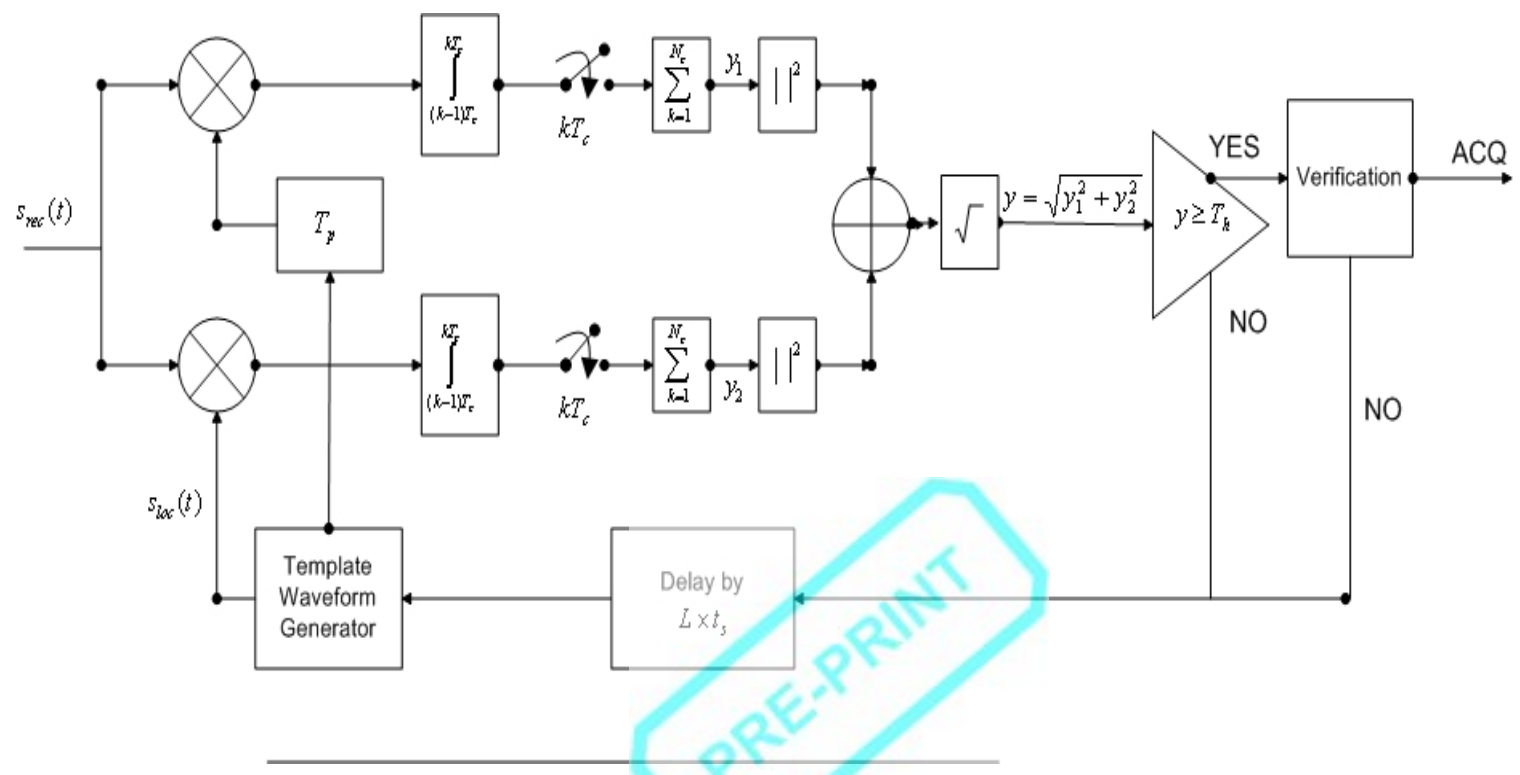

Fig. 3. Structure of an acquisition receiver employing non-consecutive search with joint twin-cell detection

using just one correlator.

\section{Performance Analysis}

In this section, we analyze the serial and random search acquisition schemes and derive expressions for their MATs. Specifically the acquisition analysis in Sections III-B and III$\mathrm{D}$ and the expressions for MAT derived therein are new to the best of our knowledge.

\section{A. Probabilities of detection and false alarm}

Owing to the presence of $L$ multipaths the uncertainty region consists of $L H_{1}$ cells and $(q-L) H_{0}$ cells. In the presence of AWGN with power spectral density $N_{0}$, the output $y$ of the correlator in the receiver structure of Fig. 2 in a $H_{0}$ cell is a zero-mean Gaussian random variable with a variance of $N_{c} N_{0}$. If we introduce a normalized threshold $\Upsilon_{t h}$ related to $T_{h}$ via $T_{h}=\Upsilon_{t h} N_{c} \sqrt{E_{c}}$, the probability of false alarm is given by

$$
P_{F A}\left(\Upsilon_{t h}\right)=\operatorname{Pr}\left(|y|>T_{h} \mid H_{0}\right)=2 Q\left(\sqrt{\frac{N_{c} E_{c}}{N_{0}}} \Upsilon_{t h}\right)
$$

On the other hand, in the $l^{t h} H_{1}$ cell, the output of the correlator is Gaussian with variance $N_{c} N_{0}$ as before, while its mean $N_{c} \alpha_{l} \sqrt{E_{c}}$ depends on the path amplitude $\alpha_{l}$. Therefore, the conditional probability of detecting the $l^{t h} H_{1}$ cell given the $l^{\text {th }}$ path amplitude is

$$
\begin{aligned}
P_{D_{l}}\left(\Upsilon_{t h}\right)= & \operatorname{Pr}\left(|y|>T_{h} \mid H_{1}\right) \\
= & Q\left(\sqrt{\frac{N_{c} E_{c}}{N_{0}}}\left(\Upsilon_{t h}-\alpha_{l}\right)\right) \\
& +Q\left(\sqrt{\frac{N_{c} E_{c}}{N_{0}}}\left(\Upsilon_{t h}+\alpha_{l}\right)\right)
\end{aligned}
$$

The false alarm and detection probabilities for the twin-cell scheme depicted in Fig. 3 are different and are shown in section III-F

\section{B. Serial Search in conventional multipath channels}

Traditional analyses of acquisition using serial search in conventional wideband multipath channels have made simplifying assumptions, namely that the number of multipath components is much smaller than the total number of cells that the acquisition receiver has to test [3], [4] or that the paths have equal powers [5]. In the following, a complete 


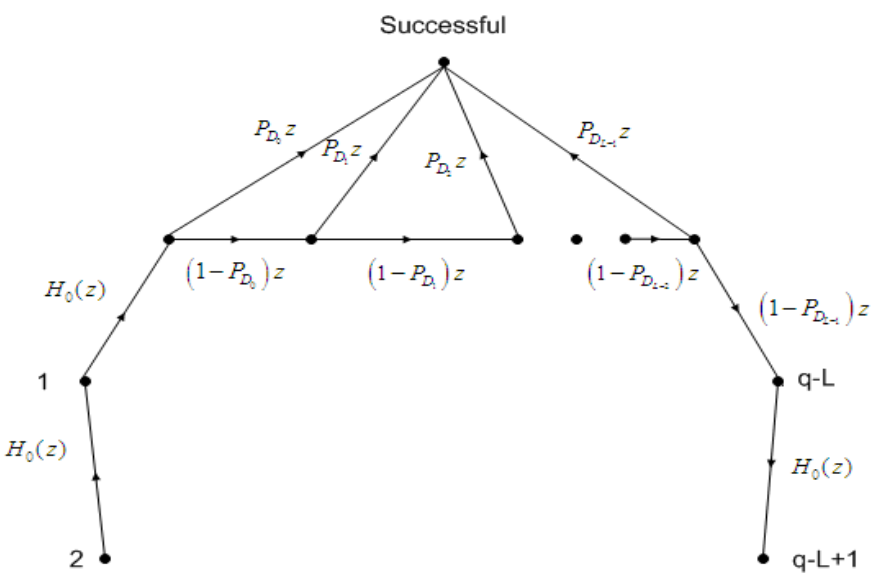

Fig. 4. Circular state diagram for the serial search acquisition system in multipath

analysis of serial search acquisition is presented without the above assumptions by invoking the transfer function approach for the acquisition time variable. We separately compute the transfer function starting from a $H_{0}\left(H_{1}\right)$ cell to the final acquisition state denoted by $A C Q$. The relevant state diagram is shown in Fig 4.

(i) Given that the search starts in a $H_{0}$ cell that is $i$ cells to the left of the first $H_{1}$ cell, the transfer function of the delay in reaching the ACQ state is given by

$$
\begin{aligned}
H_{i}(z)_{\left(H_{0}\right)}= & H_{0}^{i}(z) \times\left[H_{D}(z)+\right. \\
& \left.H_{M}(z) H_{0}^{(q-L-i)}(z) H_{i}(z)_{\left(H_{0}\right)}\right] \\
= & \frac{H_{0}^{i}(z) H_{D}(z)}{1-H_{M}(z) H_{0}^{(q-L)}(z)}
\end{aligned}
$$

where,

$$
\begin{aligned}
H_{0}(z) & =P_{F A} z^{J+1}+\left(1-P_{F A}\right) z \\
H_{D}(z) & =\sum_{j=1}^{L} P_{D_{j}} z \prod_{k=1}^{j-1}\left(1-P_{D_{k}}\right) z \\
H_{M}(z) & =\prod_{j=1}^{L}\left(1-P_{D_{j}}\right) z
\end{aligned}
$$

$H_{0}(z), H_{D}(z)$ and $H_{M}(z)$ are the transfer functions for exiting a $H_{0}$ cell, absorption into the ACQ state starting from the first $H_{1}$ cell before encountering a $H_{0}$ cell and a total miss in any round, respectively.

(ii) Given that the initial cell is the $i^{t h} H_{1}$ cell, the transfer function to reach the ACQ state is

$$
H_{i}(z)_{\left(H_{1}\right)}=H_{D_{r}}(z)+H_{M_{r}}(z) H_{q-L}(z)_{\left(H_{0}\right)}
$$

where $H_{D_{r}}(z)$ and $H_{M_{r}}(z)$ are the transfer functions denoting absorption into the ACQ state starting from the $i^{\text {th }} H_{1}$ cell before encountering a $H_{0}$ cell and missing the $H_{1}$ cells numbered $i$ through $L$, respectively.

$$
\begin{aligned}
& H_{D_{r}}(z)=\sum_{j=i}^{L} P_{D_{j}} z \prod_{k=i}^{j-1}\left(1-P_{D_{k}}\right) z \\
& H_{M_{r}}(z)=\prod_{j=i}^{L}\left(1-P_{D_{j}}\right) z
\end{aligned}
$$

Since each cell has a uniform probability of $1 / q$ of being the starting cell, the overall transfer function of the serial search is given by

$$
H(z)=\frac{1}{q}\left[\sum_{i=1}^{q-L} H_{i}(z)_{\left(H_{0}\right)}+\sum_{i=1}^{L} H_{i}(z)_{\left(H_{1}\right)}\right]
$$

The mean acquisition time can then be obtained by

$$
\bar{T}_{a c q}=\left.\frac{d H(z)}{d z}\right|_{z=1} \times \tau_{D}
$$

which (after some algebra) is given by

$$
\bar{T}_{a c q}=\left[\left(\frac{q-L}{q}\right) \bar{T}_{a c q_{0}}+\left(\frac{L}{q}\right) \bar{T}_{a c q_{1}}\right] \times \tau_{D}
$$

where, $\bar{T}_{a c q_{0}}$ and $\bar{T}_{a c q_{1}}$ are the mean number of steps that would be taken to get to the $A C Q$ state if the search were to commence from a $H_{0}$ cell or a $H_{1}$ cell respectively and are shown on the bottom of this page. In (16), $T_{D}$ is the mean number of steps to reach the $A C Q$ state starting from the first $H_{1}$ cell given that the search doesn't encounter a $H_{0}$ cell and $P_{M}$ is the probability of total miss which are given by

$$
\begin{aligned}
T_{D} & =\sum_{j=1}^{L} j P_{D_{j}} \prod_{k=1}^{j-1}\left(1-P_{D_{k}}\right) \\
P_{M} & =\prod_{k=1}^{L}\left(1-P_{D_{k}}\right)
\end{aligned}
$$

Equation $\bar{T}_{a c q_{1}}$ is essentially the formula derived in [3] where it is assumed that the search starts outside the $H_{1}$ cells. When $P_{D_{j}}=P_{D}$ for all $j$, the formula for $\bar{T}_{a c q}$ simplifies to the one derived in [5] where it is assumed that all the paths have equal powers. At high signal-to-noise ratios when

$$
\begin{aligned}
\bar{T}_{a c q_{0}}= & \frac{2 T_{D}+\left(q+q P_{M}-L+1-P_{M}\right)\left(1+J P_{f}\right)+L P_{M}\left(1-J P_{f}\right)}{2\left(1-P_{M}\right)} \\
\bar{T}_{a c q_{1}}= & \frac{1}{L}\left(\sum_{i=1}^{L} \sum_{j=i}^{L}(j-i+1) P_{D_{j}} \prod_{k=i}^{j-1}\left(1-P_{D_{k}}\right)\right) \\
& +\frac{1}{L}\left(\sum_{i=1}^{L}\left(\frac{T_{D}+(q-L)\left(1+P_{f} J\right)+L P_{M}}{\left(1-P_{M}\right)}+(L-i+1)\right)\left(\prod_{k=i}^{L}\left(1-P_{D_{k}}\right)\right)\right)
\end{aligned}
$$


$P_{F A} \rightarrow 0$ and $P_{D_{i}} \rightarrow 1 \forall i$, the process takes one integration period to reach the ACQ state if the search falls in any of the $H_{1}$ cells and $(i+1)$ integration durations if it falls in a $H_{0}$ cell that is $i$ cells removed from the nearest $H_{1}$ cell. Therefore, $\bar{T}_{a c q}$ reduces to (18), which is the same as that derived in [8].

$$
\begin{aligned}
\bar{T}_{a c q}(s a t) & =\frac{1}{q}\left[L+\sum_{i=1}^{q-L}(i+1)\right] \times \tau_{D} \\
& =\left(\frac{2(L-1)+(q-L+1)(q-L+2)}{2 q}\right) \tau_{D}
\end{aligned}
$$

\section{Serial Search in IEEE 802.15.3a multipath channels}

In contrast to the conventional wideband channel models, the UWB channel models as prescribed by the IEEE 802.15.3a do not necessarily have multipath arrivals at every tap location of the delay line. Thus, the analysis of serial search in UWB channels requires not only the knowledge of the number of multipath components and their $P_{D}$ 's, but the relative locations of all the paths for each channel realization. This implies the need for a realization dependant state diagram and resultant MAT that must be averaged; owing to this complexity we resorted to simulations to determine the mean acquisition times of serial search in UWB channels.

\section{Random Search for conventional wideband and UWB channels}

In the following we develop an analysis of random search acquisition that makes no assumption about the multipath arrivals and thus can be applied to both conventional wideband channel models as well as the IEEE 802.15.3a models.

In random search, since the $H_{1}$ cells are dispersed randomly among the $H_{0}$ cells, the overall transfer function can be derived by noting that when the process is in a $H_{0}$ cell, it has a $1-L / q$ probability of transitioning to a $H_{0}$ cell in the next step and a $1 / q$ probability of transitioning to each of the $L H_{1}$ cells. Therefore, the transfer function for exiting an $H_{0}$ cell is given by

$$
\begin{aligned}
H_{0}(z)= & \left(P_{F A} z^{J+1}+\left(1-P_{F A}\right) z\right) \times \\
& {\left[\left(\frac{q-L}{q}\right) H_{0}(z)+\left(\frac{1}{q}\right) \sum_{i=1}^{L} H_{i}(z)\right] }
\end{aligned}
$$

where $H_{i}(z)$ is the transfer function for exiting the $i^{\text {th }} H_{1}$ cell. On the other hand, when the search is in the $i^{\text {th }} H_{1}$ cell, it could move to the $A C Q$ state with a probability of $P_{D_{i}}$, to a $H_{0}$ cell with a probability of $\left(1-P_{D_{i}}\right)(1-L / q)$ and to each of the remaining $H_{1}$ cells with a probability of $\left(1-P_{D_{i}}\right)(1 / q)$. Therefore the transfer function from the $i^{t h}$ $H_{1}$ cell is given by

$$
\begin{aligned}
H_{i}(z)=P_{D_{i}} z+ & \left(1-P_{D_{i}}\right) z \times\left[\left(\frac{q-L}{q}\right) H_{0}(z)\right. \\
+ & \left.\left(\frac{1}{q}\right) \sum_{j=1}^{L} H_{j}(z)\right], \quad i=1,2 . ., L
\end{aligned}
$$

The $H_{i}(z)$ 's and $H_{0}(z)$ can be obtained by simultaneously solving the $L+1$ equations obtained from (19) and (20). Since the search has a $1-L / q$ probability of starting in a $H_{0}$ cell and a $1 / q$ probability of starting in each of the $H_{1}$ cells, the effective transfer function to reach the $A C Q$ state is given by

$$
H(z)=\left(\frac{q-L}{q}\right) H_{0}(z)+\left(\frac{1}{q}\right) \sum_{i=1}^{L} H_{i}(z)
$$

The MAT is again calculated using (14). For random search, the formula for the mean acquisition can be shown to be given by the closed form

$$
\bar{T}_{a c q}=\frac{P_{F A} J(q-L)+q}{\sum_{l=1}^{L} P_{D_{l}}} \times \tau_{D}
$$

Alternatively, the expression for MAT can be derived purely from probabilistic arguments. Since the search is memoryless, the probability of acquisition $p_{a c q}$ at any step is the probability of hitting one of the $H_{1}$ cells and detecting it.

$$
p_{a c q}=\frac{1}{q} \sum_{l=1}^{L} P_{D_{l}}
$$

The number of steps until successful acquisition is thus geometrically distributed with parameter $p_{a c q}$. If $C_{f}^{(n)}$ is the time delay incurred by $n$ unsuccessful steps, then the MAT can be determined from

$$
\bar{T}_{a c q}=\left[\sum_{n=0}^{\infty}\left(C_{f}^{(n)}+1\right)\left(1-p_{a c q}\right)^{n} p_{a c q}\right] \tau_{D}
$$

To calculate $C_{f}^{(n)}$, we note that given that there have been $n$ unsuccessful attempts, the number of times a $H_{0}$ cell has been visited follows a binomial distribution with probability

$$
\begin{aligned}
p_{H_{0}} & =\operatorname{Pr}\left\{H_{0} \text { cell } \mid \text { acq. unsuccessful }\right\} \\
& =\frac{\operatorname{Pr}\left\{\text { acq. unsuccessful } \mid H_{0} \text { cell }\right\} \operatorname{Pr}\left\{H_{0} \text { cell }\right\}}{\operatorname{Pr}\{\text { acq. unsuccessful }\}} \\
& =\frac{(q-L) / q}{1-p_{a c q}}=\frac{q-L}{q-\sum_{l=1}^{L} P_{D_{l}}}
\end{aligned}
$$

Therefore the time delay incurred due to $n$ unsuccessful steps is obtained by averaging over $i$, the number of times $H_{0}$ cells are visited.

$$
\begin{aligned}
C_{f}^{(n)}= & \sum_{i=0}^{n}\left(\left[i\left(P_{F A}(J+1)+\left(1-P_{F A}\right)\right)+(n-i)\right]\right. \\
& \left.\left(\begin{array}{c}
n \\
i
\end{array}\right) p_{H_{0}}^{i}\left(1-p_{H_{0}}\right)^{(n-i)}\right) \\
= & n\left(p_{H_{0}} P_{F A} J+1\right)
\end{aligned}
$$

Substituting (23), (25) and (26) in (24), the MAT is obtained as

$$
\bar{T}_{a c q}=\frac{P_{F A} J(q-L)+q}{\sum_{l=1}^{L} P_{D_{l}}} \times \tau_{D}
$$

In random search at high signal-to-noise ratio values, the probability of acquisition is just the probability that the search lands in one of the $H_{1}$ cells, which is $L / q$. The number of jumps to get to a $H_{1}$ cell follows a geometric distribution with a mean of $q / L$. Therefore the MAT at high signal-to-noise ratios is 


$$
\bar{T}_{a c q}(s a t)=\left(\frac{q}{L}\right) \tau_{D}
$$

which is the same as predicted by (27) for $P_{F A}=0, P_{D_{l}}=1$ $\forall l$.

\section{E. Non-consecutive Search with cell-by-cell detection in con- ventional multipath channels}

The non-consecutive search was proposed by Shin and Lee in [12], where a complete analysis is presented based on the state diagram and the formula for MAT derived. The acquisition scheme described therein involves a verification stage and the expression for the MAT has some minor typographical errors. We re-derived the formula for the case of no verification stage and corrected the typographical errors in the expression for $\bar{T}_{a c q}$, which is shown on the bottom of this page.

In (29),

$$
\begin{aligned}
H_{0}^{\prime} & =1-P_{F A}+(J+1) P_{F A} \\
H_{M} & =\prod_{l=1}^{L}\left(1-P_{D_{l}}\right) \\
H_{M}^{\prime} & =L \prod_{l=1}^{L}\left(1-P_{D_{l}}\right) \\
H_{D}(l, j) & =P_{D_{((j))}} \prod_{k=1}^{j-l}\left(1-P_{D_{((j-k))}}\right) \\
H_{D}^{\prime}(l, j) & =P_{D_{((j-l))}}(j-l+1) \prod_{k=1}^{j-l}\left(1-P_{D_{((j-k))}}\right)
\end{aligned}
$$

In the above expressions, $((l)) \triangleq[(l-1) \bmod L]+1$.

\section{F. Non-consecutive Search with joint twin-cell detection in} conventional multipath channels

The analysis of NCS-TC is the same as that of NCS-CC except that the false alarm and detection probabilities are modified due to the combining of the cells. When the two cells combined are $H_{0}$ cells, the decision variable $y$ has a Rayleigh distribution since $y_{1}$ and $y_{2}$ themselves are zero mean Gaussian random variables with variance $N_{c} N_{0}$. However, it is possible that the present cell is a $H_{0}$ cell and the previous cell is a $H_{1}$ cell. When this happens, the $H_{1}$ cell is the last multipath component, which has a small signal energy component when the exponential profile is assumed. Further this $H_{1}-H_{0}$ cell combination, which could lead to a false alarm, happens only once in $L$ cycles while the $H_{0}-H_{0}$ combination happens $(q / L-1)$ times every cycle. Therefore, the effect of $H_{1}-H_{0}$ cell combination can be neglected in typically decaying profiles and the probability of false alarm is essentially given by

$$
P_{F A}\left(\Upsilon_{t h}\right)=\operatorname{Pr}\left(y>T_{h} \mid H_{0}\right)=\exp \left(-\frac{N_{c} E_{c}}{2 N_{0}} \Upsilon_{t h}^{2}\right)
$$

However, when the cells being combined are $H_{1}$ cells, the decision variable has a Ricean distribution since $y_{1}$ and $y_{2}$ are non-zero mean Gaussian random variables. Therefore, the probabilities of detection are given by [16]

$$
P_{D_{l}}=Q_{1}\left(\sqrt{\frac{N_{c} E_{c}}{N_{0}}} \lambda_{l}, \sqrt{\frac{N_{c} E_{c}}{N_{0}}} \Upsilon_{t h}\right)
$$

where $Q_{1}(a, b)$ is the Marcum-Q function and $\lambda_{l}^{2}$ is the noncentrality parameter.

$$
\lambda_{l}=\left\{\begin{array}{cl}
\alpha_{1} & l=1 \\
\sqrt{\alpha_{l}^{2}+\alpha_{l-1}^{2}} & l=2,3, . ., L
\end{array}\right.
$$

\section{Simulations}

We simulated both the conventional wideband channel model and the IEEE 802.15.3a channel model to evaluate and compare the performances of the search schemes described in the foregoing sections. For each channel realization, knowing the channel gains, the analytical results were obtained by first calculating the probabilities of detection and false alarm from the equations shown in Sections III-A and III-F and subsequently using these probabilities in the MAT formulas derived. For the simulation results, the probabilities were first simulated and were subsequently used in the MAT simulations. In either case, the MATs were averaged over 100 channel realizations.

For our simulations we used a UWB pulse of width 1 ns and a PN code of length 128. Considering the bandwidth of this pulse $(4 \mathrm{GHz})$, the multipath resolution $t_{s}$ was taken to be $0.25 \mathrm{~ns}$, i. e. $M=4$. The total number of cells in the uncertainty region is thus $q=512$. Threshold setting was based on constant false alarm rate (CFAR) with $P_{F A}=10^{-4}$.

\section{A. Conventional wideband multpath channel model}

Fig. 5 shows the results of the simulations using the conventional wideband channel model with exponential $(\eta=0.25)$ and uniform $(\eta=0)$ power delay profiles for delay spreads of $16 \mathrm{~ns}(L=64)$ and $32 \mathrm{~ns}(L=128)$. Comparing (a) and (b) for $L=64$ and (c) and (d) for $L=128$, it is evident that random search is the preferred strategy over serial search when

$$
\begin{gathered}
\bar{T}_{a c q}=\frac{\sum_{l=1}^{L} \sum_{j=l}^{L+l-1} \frac{q}{L}\left(\sum_{k=l}^{j} \frac{q}{L}+l-j-\frac{1}{2}\left(\frac{q}{L}+1\right)\right) H_{0}^{\prime} H_{D}(l, j)+\frac{q}{L} H_{D}^{\prime}(l, j)}{L\left(1-H_{M}\right)} \times \tau_{D} \\
+\frac{\sum_{l=1}^{L} \sum_{j=l}^{L+l-1} \frac{q}{L} H_{D}(l, j)\left((q-L) H_{0}^{\prime} H_{M}+H_{M}^{\prime}\right)}{L\left(1-H_{M}\right)^{2}} \times \tau_{D}
\end{gathered}
$$


(a)

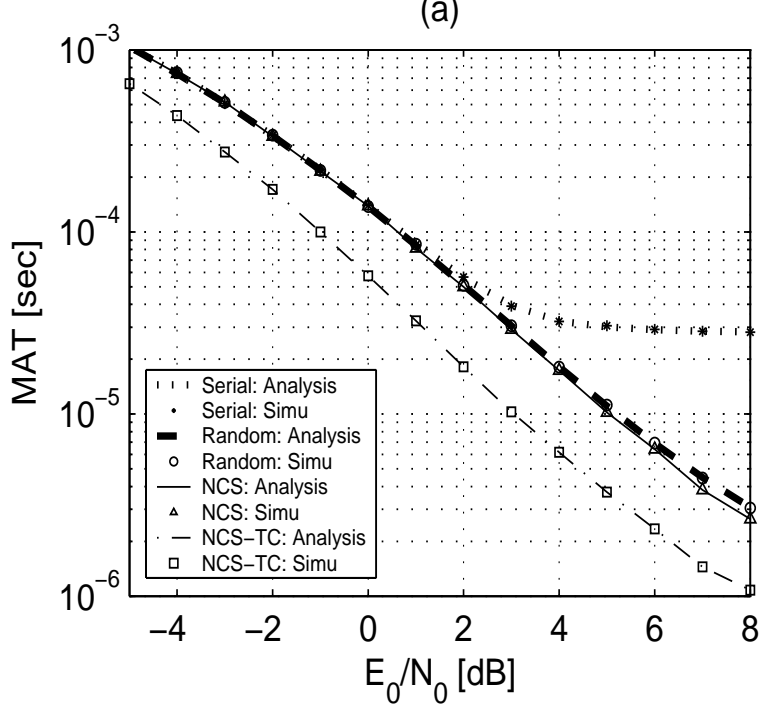

(c)

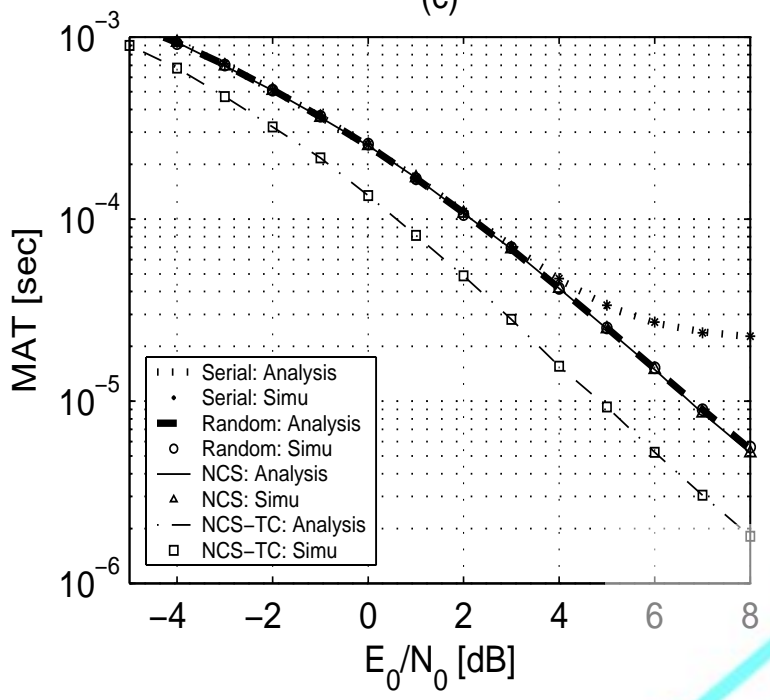

(b)

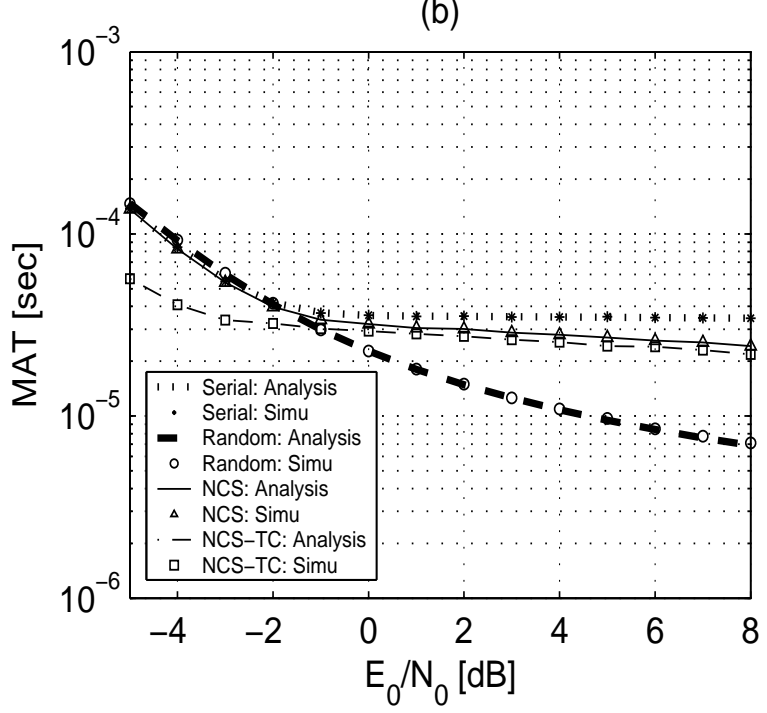

(d)

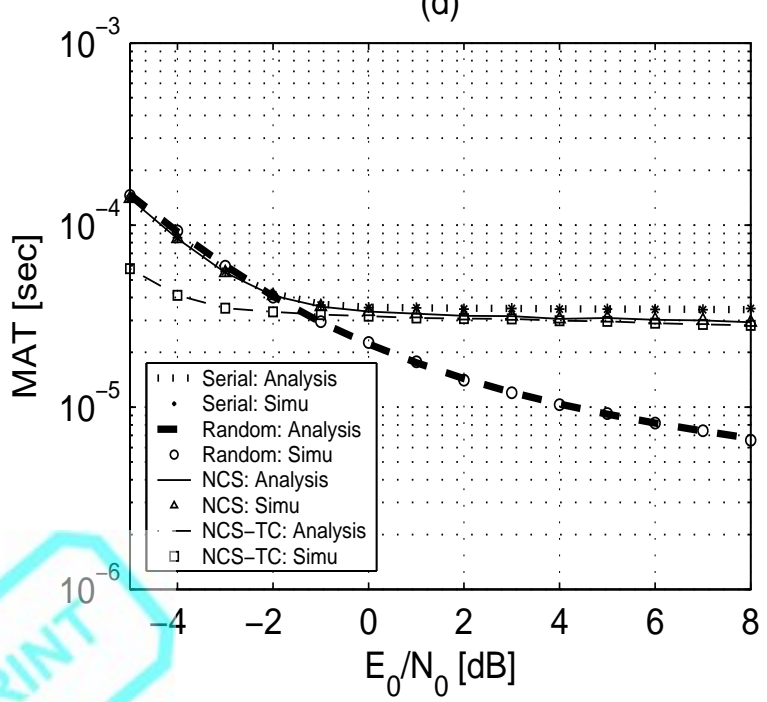

Fig. 5. Mean acquisition time in conventional wideband multipath channel models with (a) uniform PDP and $L=64$ and (b) exponential PDP and $L=64$ (c) uniform PDP and $L=128$ (d) exponential PDP and $L=128$

the multipath profile is uniform. In the uniform PDP case, while the performance of random search is the same as serial search at lower SNRs, it clearly outperforms the latter at higher SNRs. On the other hand, when the profile is exponential, serial search performs marginally better than random search at lower SNRs, but the latter quickly takes over as the SNR increases. The reason is that while random search has the advantage of being able to reach a $H_{1}$ cell faster on average when the number of paths is large, the advantage of serial search is that it encounters paths with higher probabilities of detection earlier (relative to those with lower detection probabilities) when the profile is exponential. However, serial search does not enjoy its advantage when the profile is uniform and it's performance is always worse than random search. In exponential PDP conditions, the natural advantage of random search outweighs that of serial search only beyond a certain SNR. Further, as the number of paths increases, the mean number of steps required to reach a $H_{1}$ cell decreases faster for random search than for serial search. In summary, random search gives the most benefit when the multipath profile is uniform and the number of multipath components is large.

The NCS scheme, owing to its step size, reduces the number of steps to reach a $H_{1}$ cell compared to the conventional serial search. However, at low SNR values, when the detection probabilities are low, this benefit is not apparent and so the performance improvement provided by NCS is not very significant. At high SNR values, since the $P_{D}$ values are high, the time required to get to the $H_{1}$ cells is the factor limiting MAT performance and thus the NCS scheme provides far better performance than serial search. In fact, at sufficiently high values of SNR, the acquisition times obtained by using the non-consecutive search can be up to $L$ times lower than those obtained using serial search. Like random search, the NCS scheme also gives the most benefit when the multipath 
(a)

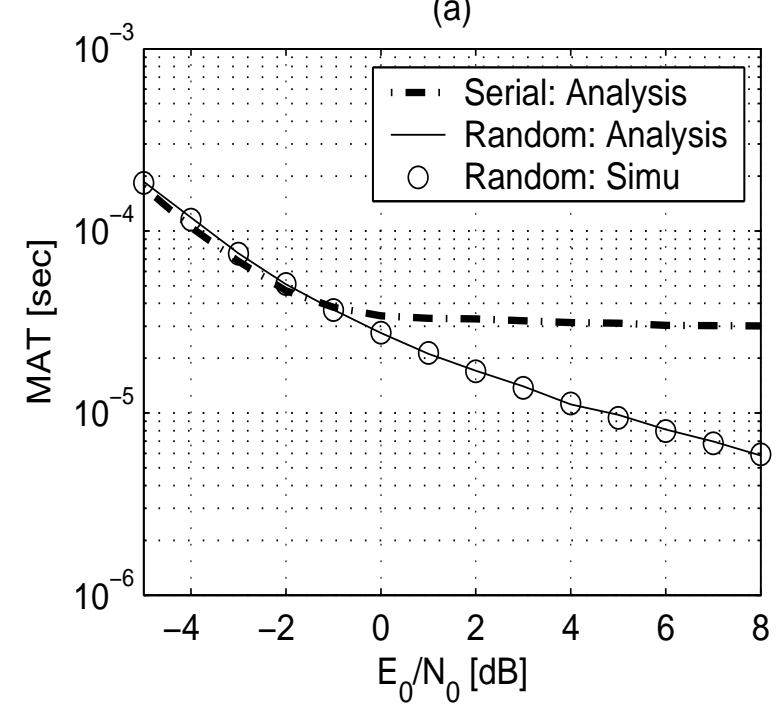

(c)

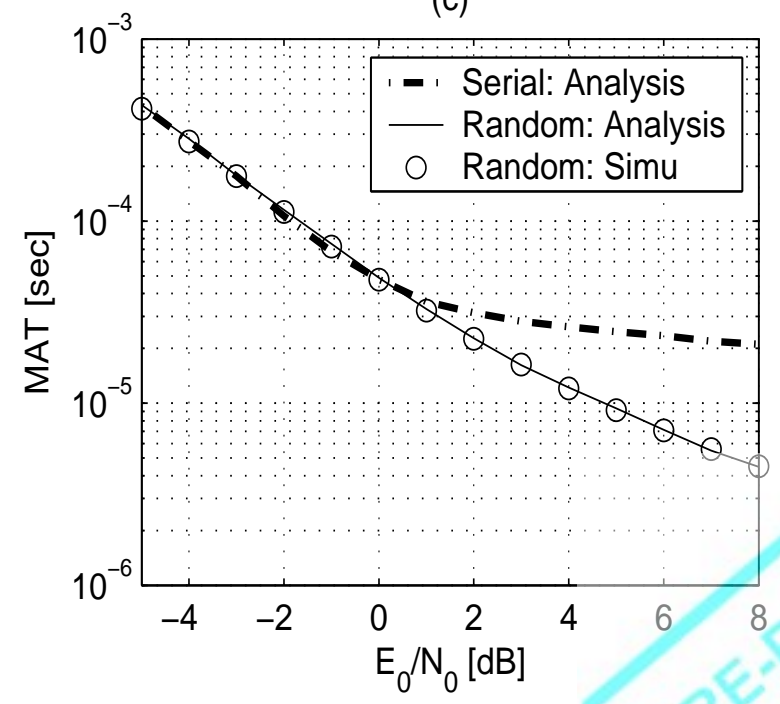

(b)

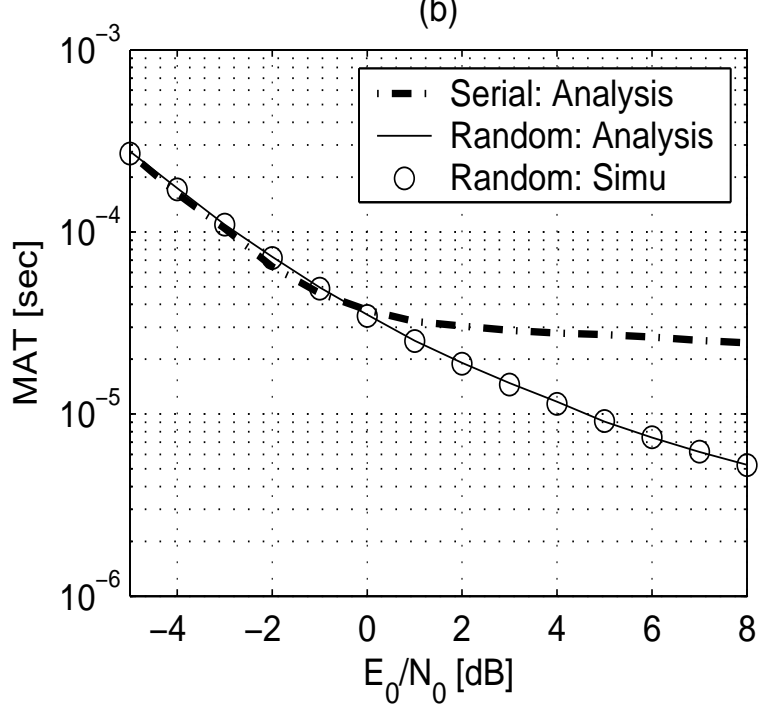

(d)

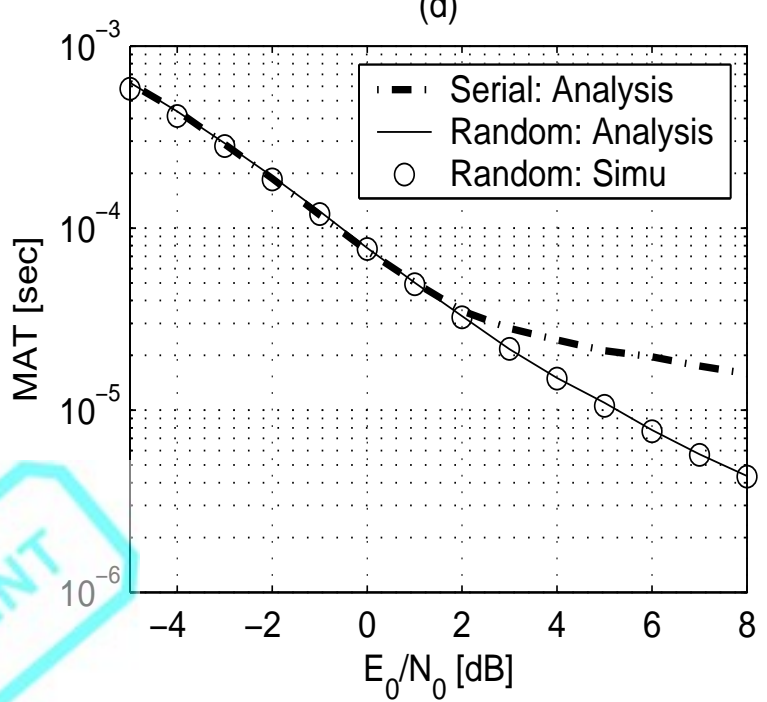

Fig. 6. Mean acquisition time in the IEEE 802.15.3a channel models (a) CM1, (b) CM2, (c) CM3 and (d) CM4

profile is uniform.

The problem of small improvements in performance at low SNR values is addressed by the NCS-TC scheme. The use of equal gain combining improves the probability of detection and consequently there is a noticeable improvement in performance at low SNR values. The MAT curve could be shifted further to the left by using more than two correlators indicating a design trade-off between hardware complexity and performance. Moreover, beyond a certain point, the number of correlators results in diminishing returns, particularly if the channel intensity profile is exponential in nature.

\section{B. UWB channel models}

The NCS-CC and the NCS-TC acquisition schemes are designed to exploit the presence of $L$ contiguous multipath components by having a step size $L$ times larger than serial search. The IEEE 801.15.3a model however does not assume presence of multipath components at contiguous tap locations.
Hence the non-consecutive schemes cannot be applied to UWB channels. Furthermore, the NCS and the NCS-TC schemes need knowledge of the number of multipath components, which requirement restricts its application. Considering these constraints, we restrict our comparisons in 802.15 .3a channel models to only the serial and random search schemes.

For our simulations, we considered 100 realizations of channel models 1 through 4 (CM1, CM2, CM3 and CM4) defined by IEEE $802.15 .3 \mathrm{a}$ [6] with a resolution of $0.25 \mathrm{~ns}$. The results of the simulations are plotted in Fig. 6, which shows the acquisition times as a function of SNR for the two acquisition schemes - the conventional serial search and random search schemes.

It is clear from the figure that the performance advantage of using random search increases with SNR as is the case with conventional multipath channels and for the same reasons. The most striking observation however is that although the performance of serial search is marginally better at lower 
SNRs, this difference diminishes as we move to higher channel models which are characterized by more random multipath arrivals and longer delay spreads. In fact, as we move from CM1 to CM4, the performance of serial search approaches that of random search at all SNR values.

The reason for this behavior is that the inherent randomness of the multipath arrivals in the higher channel models of 802.15.3a renders the behavior of serial search to be very similar to random search. Even in CM4, the multipath arrivals are not completely random in that they are still clustered, which explains the little difference in performance between the serial and random searches. As the multipath arrivals become more random, i. e. the $H_{1}$ cells become more uniformly dispersed among $H_{0}$ cells, the performance of serial search scheme - or any deterministic search scheme for that matter would become indistinguishable from that of random search. This underscores the broad utility of the random search analysis developed - it can be used as a good model for any deterministic search scheme where the multipath arrivals are 'randomized'.

\section{Conclusions}

We have provided an accurate analysis of serial search acquisition by removing the simplifying assumptions invoked for conventional DS-SS systems that are not valid for wideband systems employing short PN codes. We have also derived an expression for the MAT for random search that is applicable in any general multipath channel model. It has been shown that in conventional wideband channel models, the random search provides significant improvement in performances over serial search when the number of multipath components is large and especially when the multipath profile is more uniform. Several indoor channel models like those used in UWB tend to have random multipath arrivals. It has been shown that in such channels, the random search analysis developed can be used to model any deterministic search scheme.

\section{REFERENCES}

[1] A. Polydoros and C. Weber, "A unified approach to serial search spread-spectrum code acquisition-Part I: General theory," in IEEE Trans Commun, vol. 32, no. 5, pp. 542-549, May 1984.

[2] J. K. Holmes and C. C. Chen, "Acquisition time performance of PN spread-spectrum systems," in IEEE Trans Commun, vol. 25, no. 8, pp. 778-784, Aug 1977.

[3] L. L. Yang and L. Hanzo, "Serial acquisition of DS-CDMA signals in multipath fading mobile channels," in IEEE Trans. Veh. Technol., vol. 50, no. 2, pp. 617-628, Mar 2001.

[4] B. B. Ibrahim and A. H. Aghvami, "Direct sequence spread spectrum matched filter acquisition in frequency-selective Rayleigh fading channels," in IEEE J. Select. Areas Commun., vol. 12, pp. 885-890, June 1994.

[5] J. Iinatti, "Mean acquisition time of DS code synchronization in fixed multipath channel," in IEEE International Symposium on Spread Spectrum Techniques and Applications, vol. 1, pp. 116-120, Sept 1998.

[6] J. R. Foester et. al., "Channel modeling sub-committee report final,", tech. rep., IEEE P802.15 Wireless Personal Area Networks, P802.1502/490r1-SG3a, Feb. 2003.

[7] A. F. Molisch, J. R. Foerster, and M. Pendergrass, "Channel models for ultrawideband personal area networks," in IEEE Wireless Communications, pp. 14-21, Dec 2003.

[8] E. A. Homier and R. A. Scholtz, "Rapid acquisition of ultra-wideband signals in the dense multipath channel," in IEEE Conference on Ultra Wideband Systems and Technologies (UWBST), pp. 105-109, May 2002.
[9] M. Z. Win and R. A. Scholtz, "Impulse radio: How it works," in IEEE Communication Letters, vol. 2, pp. 36-38, Feb 1998.

[10] K. Siwiak, "Ultra-wideband radio: Introducing a new technology," in Proc. 2001 Spring Vehicular Technology Conf., pp. 1088-1093, 2001.

[11] S. Roy, J. R. Foerster, V. Somayazulu, and D. G. Leeper, "Ultrawideband radio design: The promise of high-speed, short-range wireless connectivity," in Proc. of the IEEE. Special Issue on Gigabit Wireless, vol. 92, no. 2, pp. 292-311, Feb 2004.

[12] O. S. Shin and K. B. Lee, "Utilization of multipaths for spread-spectrum code acquisition in frequency-selective rayleigh fading channels," in IEEE Trans. Communication, vol. 49, pp. 734-743, Apr 2001.

[13] R. Roberts, "Xtremespectrum CFP document." http://grouper.ieee.org/groups / $802 / 15 / \mathrm{pub} / 2003 / \mathrm{Jul} 03 / 03154$ r3P802-15_ TG3a-XtremeSpectrum-CFP-Documentation.pdf.

[14] A. Saleh and R. Valenzuela, "A statistical model for indoor multipath propagation," in IEEE J. on Sel. Areas in Comm., vol. SAC-5, no. 2, pp. 128-137, Feb 1987.

[15] A. J. Viterbi, CDMA: Principles of spread spectrum communication. MA, U. S. A: Addison-Wesley, 1995.

[16] J. G. Proakis, Digital Communications. NY, U. S. A: McGraw-Hill, 1989.

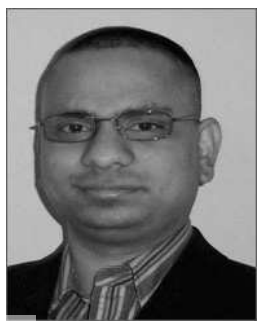

Iyappan Ramachandran (S'04) received the B. E. (Hons.) degree from Birla Institute of Technology and Science, Pilani, India and the M. S. degree from the University of Washington, Seattle both in electrical engineering in 2000 and 2004 respectively. Since April 2004, he has been working toward his $\mathrm{Ph}$. D degree in electrical engineering at the University of Washington, Seattle.

His main research interests are UWB systems, acquisition/synchronization issues of wideband systems, localization and positioning, MAC design for ad-hoc wireless systems and PHY-MAC cross-layer optimization. At present, his focus is on the design of energy-efficient PHY and MAC principles for wireless sensor networks.

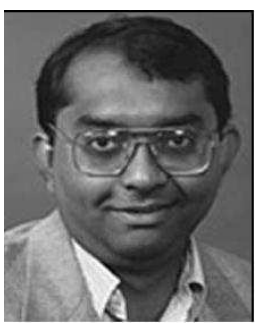

Sumit Roy (S'84-M'88-SM'00) received the B.Tech. degree from the Indian Institute of Technology, Kanpur, in 1983, and the M.S. and Ph.D. degrees from the University of California, Santa Barbara, all in electrical engineering, in 1985 and 1988, respectively, as well as the M.A. degree in statistics and applied probability in 1988.

His previous academic appointments were at the Moore School of Electrical Engineering, University of Pennsylvania, and at the University of Texas, San Antonio. He is presently a Professor of electrical engineering at the University of Washington, where his research interests center around analysis/design of physical and data link layer of communication systems, with a topical emphasis on next-generation mobile/wireless networks. He is an Editor for the Wiley Journal on Wireless Communications and Mobile Computing.

Dr. Roy is an Editor for the IEEE TRANSACTIONS ON WIRELESS COMMUNICATIONS. His activities for the IEEE Communications Society includes membership of several technical committees and technical program committees for conferences. 